Jorge Arturo Salas Plata Mendoza. Estudió Ingeniería Civil en la Universidad Autónoma de Chihuahua, donde se graduó en el año de 1976. Posteriormente estudió la Maestría en Ingeniería de los Aprovechamientos Hidráulicos en la División de Estudios de Posgrado de la Facultad de Ingeniería de la Universidad Nacional Autónoma de México. Además, realizó estudios de Maestría en Planeación y Administración de los Recursos Hidráulicos en el Departamento de Geografía de la Universidad del Sur del Estado de Illinois en EE.UU. Finalmente llevó a cabo estudios de Doctorado en Ingeniería y Ciencias del Medio Ambiente en Centro de Administración de los Recursos Ambientales de la Universidad de Texas en El Paso, EE.UU. Trabajó durante 18 años para el sector público en lo que actualmente es la Comisión Nacional del Agua. Actualmente es Profesor de Tiempo Completo en el Departamento de Ingeniería Civil y Ambiental del Instituto de Ingeniería y Tecnología de la Universidad Autónoma de Ciudad Juárez y pertenece al Cuerpo Académico de Estudios del Agua.

Thelma García. Cursó la Licenciatura en Ciencias de la Información en la Universidad Autónoma de Chihuahua, estudió la Maestría en Bibliotecología y Ciencias de la Información en el Instituto Tecnológico de Monterrey y en el año 2016 obtuvo el grado de Doctora en Pedagogía por la Universidad de Ciencias Pedagógicas "Enrique José Varona”, en La Habana, Cuba. De 1999 a 2011 laboró en la biblioteca del Tecnológico de Monterrey Campus Chihuahua y posteriormente fue directora del Departamento de Lenguaje y Comunicación en la Preparatoria itesm hasta 2014. Asimismo, ha sido docente en la Universidad Autónoma de Chihuahua, donde a lo largo de cinco años impartió clases para la carrera de Ciencias de la Información. Del año 2007 al 2008 fue becaria del Instituto Chihuahuense de la Cultura y del Consejo Nacional para la Cultura y las Artes. Cuenta con diversas publicaciones nacionales e internacionales desde el año 2014 a la fecha, es Profesora-Investigadora de Tiempo Completo y miembro del Cuerpo Académico Competencias Informacionales para la Vida Académica y la Ciudadanía Digital (UACJ-CA-I 22 ) en la Universidad Autónoma de Ciudad Juárez.

Historial editorial Recepción: 18 de febrero de 2021 Revisión: 27 de febrero de 2021 Aceptación: 18 de abril de 2021 Publicación: 28 de junio de 2021 


\title{
Grupo Retos del 2030 en la UACJ: sentido de pertenencia, salud y formación medioambiental del estudiantado
}

\section{The 2030 Challenges Group at UACJ: \\ sense of ownership, health and \\ environmental education of the students \\ Grupo Desafios de 2030 na UACJ: sentimento de pertencimento, saúde e formação ambiental dos alunos}

\author{
Jorge Arturo Salas Plata Mendoza y Thelma García \\ Universidad Autónoma de Ciudad Juárez \\ jsalas@uacj.mx/thelma.garcia@uacj.mx
}

\section{Resumen}

El sentido de pertenencia y la salud de los jóvenes estudiantes se ha visto comprometido, así como su valoración de la situación actual en el contexto de la llamada crisis de civilización. El Grupo Retos del 2030 se concibió durante un curso de escritura dialógica para docentes en el año 2016, en la Universidad Autónoma de Ciudad Juárez (UACJ), en Chihuahua, México, como una aportación ante los imperiosos desafíos ecológicos, de salud y educación al que se enfrenta la comunidad universitaria. La metodología consiste en el envío semanal de una lectura para su discusión; las aportaciones escritas por parte de los estudiantes al conjunto del grupo y, por último, la retroalimentación de los docentes a cada alumno con relación a la mejora de la estructura gramatical y redacción. El grupo en la actualidad integra una comunidad de 631 personas, en su gran mayoría estudiantes. Hasta el 2020 se había enviado semanalmente una lectura, para un total de 100 , en la que en promedio escriben 30 alumnos por semestre que reciben retroalimentación, dando un total de 3,000 revisiones. En octubre del 2020, se realizó el Primer Foro Grupo Retos del 2030 a través de la plataforma TEAMs con ponencias de los alumnos.

Palabras clave: Lectura, Escritura, Sentido de pertenencia, Salud, Medio ambiente 


\section{Abstract}

The sense of ownership and health of young students has been compromised, as well as their perception of the current situation in the context of the so-called crisis of civilization. The 2030 Challenges Group was conceived during a dialogic writing course for teachers in 2016, at the Autonomous University of Ciudad Juárez (UACJ), Chihuahua, Mexico, as a contribution to face the pressing ecological, health and education challenges to which faces the university community. The methodology consists of sending a weekly reading for discussion; then the written contributions by the students to the group as a whole and, finally, the feedback from the teachers to each student regarding the improvement of their grammatical structure and writing. The group, mainly students, currently integrates a community of 631 people. Until 2020, a weekly reading had been sent, for a total of 100, in which on average 30 students write per semester who receive feedback, giving a total of 3,000 reviews. In October 2020, the First 2030 Challenges Group Forum was held through the TEAMs platform with presentations by the students.

\section{Keywords: Reading, Writing, Sense of ownership, Health, Environ- ment}

\section{RESUMO}

O sentimento de pertença e a saúde dos jovens estudantes tem sido comprometidos, bem como a sua avaliação da situação atual no contexto da chamada crise de civilização. O grupo foi concebido durante um curso de redação dialógica para professores em 2016, na Universidade Autônoma de Ciudad Juárez (uacj), em Chihuahua, México, como uma contribuição aos urgentes desafios ecológicos, de saúde e educação que enfrenta a comunidade universitária. A metodologia consiste no envio de uma leitura semanal para discussão; as contribuições escritas dos alunos para o grupo como um todo e, por fim, o feedback dos professores para cada aluno em relação ao aprimoramento da estrutura gramatical e escrita. $\mathrm{O}$ grupo integra atualmente uma comunidade de 631 pessoas, a grande maioria estudantes. Até 2020, havia sido enviada uma leitura semanal, para um total de 100 , em que escrevem em média 30 alunos por semestre que recebem feedback, totalizando 3.000 avaliações. Em outubro de 2020, foi realizado o Primeiro Fórum de Grupos de Desafios de 2030 por meio da plataforma de equipes com apresentações dos alunos.

\section{Palavras-chave: Leitura, Escrita, Sentido de pertencimento, Saúde, Meio Ambiente}




\section{INTRODUCCIón}

El Grupo Retos del 2030 (GR2030) se gesta en un curso para docentes en el año 2016 en la Universidad Autónoma de Ciudad Juárez (UACJ), en Chihuahua, México, como una aportación ante los imperiosos retos ecológicos, de salud y educación al que se enfrenta la comunidad universitaria y el mundo en general. Estos desafíos se agudizan en la juventud juarense que ha pasado por una serie de eventos críticos tales como la guerra contra el narcotráfico durante el sexenio de $\mathrm{Fe}$ lipe Calderón y las funestas consecuencias que al día de hoy siguen latentes en la sociedad como la descomposición del tejido social, el feminicidio y la violencia. Aunado a esto, se viven los estragos de la pandemia por el SARS-CoV2.

La creación de un grupo académico extra clase dentro del marco institucional de la UACJ tuvo como finalidad que se involucraran estudiantes y docentes, así como impulsar el pensamiento crítico, la consciencia ecológica, y la discusión del manejo de la información que en las redes sociales y los medios de comunicación masivos oscilan entre la posverdad y la desinformación. Este grupo inicialmente se conformó con docentes del programa de Ingeniería Civil y Ambiental en el Instituto de Ingeniería y Tecnología (IIT) de la UACJ.

En el año 2017 se incorporaron grupos de alumnos de los diversos programas de la División Multidisciplinaria de Ciudad Universitaria (DMCU) así como personal administrativo de la Subdirección de Universidad Saludable. En el año 2019 la labor de retroalimentación de los docentes del GR2030 se reconoció por el Programa Integral de Tutorías y Trayectorias Académicas (PITTA). En ese mismo año, durante la Semana de Ingeniería del IIT, el GR2030 se presentó formalmente ante la comunidad universitaria y en mayo del 2020 se inscribió ante la Coordinación de Apoyo del Desarrollo de la Investigación y Posgrado con el título "El sentido de pertenencia, lectura y escritura en el estudiantado de la UACJ como factores para enfrentar desafíos ambientales". Asimismo, se llevó a cabo el Primer Foro del grupo con la participación de alumnos del IIT y la DMcu.

Los tres grandes ejes de trabajo académico que sustentan al GR2030 son: la crisis ecológica y medioambiental; la salud humana; y la educación.

La dinámica del GR2030 consiste en el envío semanal durante el semestre académico de un artículo relevante en uno de los temas anteriormente mencionados para su lectura y discusión. Los alumnos envían sus comentarios escritos y los docentes hacen llegar a cada uno de los participantes una retroalimentación con relación a la estructura gramatical y la ortografía de sus escritos. 
Los objetivos específicos y metodológicos del GR2030 se resumen en los siguiente:

- Organizar al estudiantado de licenciatura en grupos académicos para reforzar su sentido de pertenencia a una institución de educación superior y a su propia generación.

- Fomentar entre los estudiantes de pregrado de la UACJ la lectura con base en escritos relevantes de diversas temáticas.

- Fomentar la discusión y el pensamiento crítico entre los integrantes del GR2030.

Este artículo aborda los objetivos anteriormente mencionados, como atributos del estudiantado de la UACJ para enfrentar los desafíos medioambientales y mejorar su desempeño académico. De igual manera, se explica y analizan los desafíos medioambientales.

\section{Desarrollo}

La pandemia por el SARS-CoV2 constituye la crisis más reciente que ha enfrentado el mundo, pero no será la última a menos que los humanos moderemos las presiones que ejercemos sobre el planeta. Así lo señala el recién publicado informe del Programa de las Naciones Unidas para el Desarrollo (PNUD) que incluye un nuevo índice experimental sobre progreso humano en el que se integran las emisiones de dióxido de carbono y la huella material de los países, una medida de la extracción de materias primas en el mundo para cubrir la demanda nacional. (Programa de las Naciones Unidas para el Desarrollo, 2020).

La crisis ecológica amenaza los ecosistemas y la supervivencia de los seres vivos que habitan el planeta, debido en primera instancia, al cambio climático que pone "en relieve otros aspectos igualmente significativos, tal [como] la pérdida de biodiversidad, acidificación de los océanos, desforestación, agotamiento y contaminación de recursos hídricos, empobrecimiento de los suelos, etc.” (Soriano, 2017). Esta realidad afecta visiblemente a la juventud universitaria que encuentra un planeta impactado por el agotamiento de los recursos naturales, la falta de oportunidades y escenarios para el bienestar en general en los que se ven comprometidos su sentido de pertenencia, salud y futuro. Específicamente el sentido de pertenencia de la juventud estudiosa se complica por las noticias que dan cuenta de un mundo que se extingue y que rompe los lazos tradicionales de la convivencia social cotidiana y la solidaridad. Las migraciones humanas, como una ruptura del apego de las comunidades a sus lugares de origen, a su cultura y seguridad que se trastoca por la violencia institucional, las epidemias, la falta de oportunidades para el desarrollo integral, las crisis ecológicas 
y medioambientales, se le presentan a la juventud estudiosa en medio de un incesante flujo de información e imágenes televisivas.

Los medios de comunicación juegan un rol fundamental, para bien o para mal, en la formación de los jóvenes universitarios. Estos medios distorsionan con frecuencia la realidad en función de criterios comerciales y políticos. La omnipresencia de dichos medios en el contexto de toda una revolución tecnológica proveniente de las ciencias de la información, junto con otras variables y escenarios socioeconómicos diversos, influye en el desarrollo psicológico de la juventud estudiosa. En el plano intelectual los individuos suelen tornarse pasivos, carentes de análisis crítico y reproductores de las opiniones vertidas en las redes sociales en gran medida superficiales que obedecen a un enfoque pragmático y reduccionista que empobrecen la autonomía de juicio y creatividad de los jóvenes. Por otra parte, cuando el individuo genera una opinión propia, esta deriva de lecturas discontinuas y de textos fragmentados que no abonan a la interioridad y la reflexión. La discusión en las redes sociales a las que acuden los jóvenes universitarios seguido constituye una esgrima de opiniones, descalificaciones y de puntos de vista sin mayor fundamentación, derivadas del sentido común, el pragmatismo o la defensa a ultranza de sus argumentos. Lo anterior le significa al estudiante pérdida de tiempo, un pobre y antieconómico uso de la red de internet, y un ejercicio intelectual carente de sentido crítico, imaginación y enriquecimiento de su acervo lingüístico.

Como resultado de la falta de lectura, los jóvenes universitarios en muchas ocasiones se ven imposibilitados de compartir sus puntos de vista en forma escrita. Aunado a esta carencia, tampoco son conscientes de las funciones comunicativas de la escritura y la habilidad para pensar en el auditorio o audiencia a la que se destina el mensaje o contenido del texto, por lo que, generalmente, no son capaces de escribir y producir discursos bien estructurados, lógicos y originales sobre los contenidos de las materias de sus planes de estudio, tesis, o cualquier tema de interés. Desafortunadamente el sistema educativo mexicano no promueve la escritura y menos aún la investigación, generando así un estudiante "minusválido de la comunicación escrita [y] de profesionistas ágrafos” (Garza, 2001).

Por lo anterior, son importantes los proyectos sociales y académicos encaminados hacia la lectura de textos de calidad; lecturas sobre temas trascendentales y diversos que contribuyan a la formación de ciudadanía sobre bases éticas que nutran la comprensión humana y la solidaridad, la imaginación, la reflexión y el sentido de pertenencia y que estimulen la discusión como medio fundamental para la comunicación asertiva. 
Si el sentido de pertenencia supone para el ser humano el poder identificarse con sus pares en valores y costumbres, la ruptura del tejido social apunta en sentido contrario, de ahí que reestablecer dicho tejido, generando espacios de convivencia e identidad, son fundamentales para el desarrollo sano de la juventud. En un escenario donde la presencia universal informativa del mensaje a distancia con las noticias no corroboradas (fake news), la lectura es, entre otras actividades, un excelente principio para reconquistar el sentido de pertenencia, y la discusión responsable y fundamentada.

De modo similar, el desarrollo de la lectura debe ir mucho más allá del primer estadio que se limita a una sola decodificación de signos y significados. Es necesario poder valorar, evaluar y reflexionar un texto escrito y sobre todo integrarlo para poder realizar una interpretación sustentada del mismo. La visión de Freire (1921-1997) es contundente, no basta con poner a leer y escribir a los estudiantes solamente, también hay que darles las pautas para que ellos puedan, leer el mundo y la palabra, enlazar el lenguaje, el pensamiento y la realidad "cuya transformación, al exigir nuevas formas de comprensión, plantea también la necesidad de nuevas formas de expresión” (Freire, 1997).

La lectura crítica se vuelve imprescindible en la selva de la desinformación y la posverdad, pues ésta dará al joven herramientas para que valore, reflexione, y evalúe el origen y la tendencia ideológica de la misma. Por otra parte, "la práctica de la lectura desarrolla la capacidad de observación, atención, concentración, análisis y espíritu crítico, además de generar reflexión y diálogo" (MÉxico: Secretaría de Educación Pública, 2010). Se trata de que el joven establezca hábitos lectores que eleven su calidad académica y más aún, que con la lectura construya, de acuerdo con Arguelles, a una ciudadanía más apta, más inteligente, más consciente de su realidad, más plena en sus capacidades y aptitudes frente a muchas cosas, incluso frente a los libros fetiches de hoy por hoy, y de hace varios siglos (Arguelles, 2011).

Por último, hablando de una perspectiva pragmática, pero también desde un enfoque social, la lectura genera un bienestar y cohesión social tan importantes como los rendimientos económicos y del mercado laboral (EsPaña: Ministerio de Educación, 2010). Al acostumbrarse a las lecturas cuyo lenguaje y contenidos enriquezcan a los estudiantes universitarios, se promoverán las condiciones para cultivar la escritura significativa, y a contribuir a superar la condición ágrafa de nuestra sociedad. Más aún en México donde el índice de lectura es de sólo 3.3 libros per cápita al año de acuerdo con el INEGI, (INEGi, 2019).

La escritura, por otro lado, requiere del escritor altas habilidades intelectuales obtenidas en un largo camino de práctica y re- 
flexión del acto (Garza Almanza, 2001). Para lograr que los estudiantes elaboren escritos lógicos, generando conocimientos y puntos de vista propios sobre el tema en cuestión, es necesario que los profesores reflexionen acerca de la necesidad de que los estudiantes escriban de manera cotidiana. Se requiere, entre otras cosas, de espacios y proyectos que involucren la planeación de artículos para su discusión, y la posterior redacción de escritos por parte de los estudiantes sobre la base de dichos artículos, así como de la revisión de sus textos por parte de los profesores.

\section{Conclusiones}

E1 GR2030 en la actualidad integra una comunidad de 631 personas en su gran mayoría estudiantes. Durante el periodo 2016-2020 se ha discutido, en promedio, 100 lecturas y se han retroalimentado 3000 escritos de los estudiantes.

Como producto de estas actividades se llevó a cabo, en octubre del 2020, el Primer Foro GR2030 por medio de la plataforma TEAMS en la UACJ con la participación de alumnos, quienes expusieron sus preocupaciones en torno al deterioro ecológico, la diversidad sexual y la pandemia.

El sentido de pertenencia y la salud de los jóvenes estudiantes se ha visto comprometida, así como su valoración de la situación actual en el contexto de la llamada crisis de civilización en general y de la pandemia en particular. Esta limitación de la juventud se ve reflejada en sus escritos del GR2030 en los que, si bien lo hacen con cierta facilidad por el entrenamiento en las redes sociales, el mensaje que transmiten es de cierta desilusión y escepticismo. Aunado a lo anterior, la juventud estudiosa participante en el proyecto GR2030 manifiestan una cierta filosofía idealista en el sentido de que somos los individuos los responsables de la actual crisis por nuestras malas actitudes en relación a la salud personal y la contaminación del entorno, dejando fuera de la crítica a los grandes poderes fácticos responsables en mayor medida de los desequilibrios medioambientales.

El agotamiento de los recursos naturales advertido por los autores del informa al Club de Roma, ha traído como consecuencia que en el primer decenio del presente siglo la humanidad ya sobrepasó la capacidad de carga de los ecosistemas planetarios (Ecoavantis, 2012). Lo anterior significa que las extracciones de materiales provenientes de la naturaleza se realizan a una tasa mayor que los ritmos naturales de renovación de dichos recursos. La pandemia por el SARS-CoV2 es el resultado, a final de cuentas, de dicho desequilibrio. El sentido 
de pertenencia, y la salud de los jóvenes estudiantes se ha visto comprometida, así como su valoración de la situación actual en el contexto de la llamada crisis civilizatoria. E1 Grupo Retos del 2030 de la UACJ es una respuesta positiva para contrarrestar la ruptura del tejido social entre los jóvenes, así como un foro excelente para la lectura de escritos relevantes en materia de ecología, medioambiente, salud pública y educación superior. Así mismo, es un buen escenario para la escritura y la discusión escrita.

\section{REFERENCIAS}

Arguelles, Juan Domingo. "Sociedad, realidad y lectura". En Revista Milenio México (2011): 63.

Ecoavantis. "La humanidad sobrepasa la capacidad de carga de la tierra para 2012”. 28 de agosto de 2012. <https://www.ecoavantis.com/ la-humanidad-sobrepasa-la-capacidad-carga-la-tierra-2012/>.

España: Ministerio de Educación. "La lectura en PISA 2009: Marcos y pruebas de la evaluación”. España: Ministerio de Educación, 2010. $<$ http://www.mecd.gob.es/dctm/ievaluaci $>$.

Freire, Paulo. La importancia de leer y el proceso de liberación. México: Siglo Veintiuno Editores, 1997.

Garza Almanza, Victoriano. "La influencia de Cantinflas y Cervantes entre los profesionistas, la comunicación escrita en México". En La Jornada (2001). <https://www.jornada.com.mx/2001/07/09/cien-almanza.html>.

INEgi. "Población lectora en México con tendencia decreciente". 23 de abril de 2019. <https://www.inegi.org.mx/contenidos/saladeprensa/boletines/2019/EstSociodemo/MOLEC2018_04.pdf>.

MÉxico: Secretaría de Educación Pública. “Importancia de la lectura”. 2010. $<$ http://www.leer.sep.gob.mx/>.

Programa de las Naciones Unidas para el Desarrollo. "Profundas fracturas sociales ponen a las personas y al planeta en riesgo de colisión, según el PNUD”. 15 de diciembre de 2020. <https://www.undp.org/ content/undp/es/home/news-centre/news/2020/Human_Development_Report_2020.html>.

Soriano Clemente, Carles. “Antropoceno, crisis ecológica”. En Razón y revolución: teoría, historia, política, 30 (2017): 153-169. <https://dialnet.unirioja.es/servlet/articulo?codigo=7312400>. 